From the Developmental Therapeutics Program of the Abramson Cancer Center and Departments of Medicine, Biostatistics and Epidemiology, Otorhinolaryngology: Head and Neck Surgery, and Radiology, University of Pennsylvania, Philadelphia, PA

Submitted January 24, 2008; accepted April 6, 2008; published online ahead of print at www.jco.org on June 9, 2008.

Supported by the Templeton Family Cancer Research Fund, the Damon Runyon Cancer Research Foundation (Cl-25-05; M.S.B.), and Grants No. 1K08CA090431 (M.S.B.) and K12-CA076931 (V.G.-A.) from the National Institutes of Health/National Cancer Institute.

Authors' disclosures of potential conflicts of interest and author contributions are found at the end of this article.

Clinical Trials repository link available on JCO.org.

Corresponding author: Marcia S. Brose MD, PhD, The University of Pennsylvania, Abramson Cancer Center, Department of Otorhinolaryngology: Head and Neck Surgery, Department of Medicine, Division of Hematology/Oncology, Clinical Research Building, Room 127, 415 Curie Blvd, Philadelphia, PA 19104; e-mail: brosem@mail.med.upenn.edu.

(C) 2008 by American Society of Clinical Oncology

0732-183X/08/2629-4714/\$20.00

DOI: $10.1200 / J C O .2008 .16 .3279$

\title{
Phase II Trial of Sorafenib in Advanced Thyroid Cancer
}

Vandana Gupta-Abramson, Andrea B. Troxel, Anoma Nellore, Kanchan Puttaswamy, Maryann Redlinger, Kathy Ransone, Susan J. Mandel, Keith T. Flaherty, Laurie A. Loevner, Peter J. O'Dwyer, and Marcia S. Brose

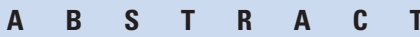

\section{Purpose}

Given the molecular pathophysiology of thyroid cancer and the spectrum of kinases inhibited by sorafenib, including Raf kinase, vascular endothelial growth factor receptors, platelet-derived growth factor receptor, and RET tyrosine kinases, we conducted an open-label phase II trial to determine the efficacy of sorafenib in patients with advanced thyroid carcinoma.

\section{Patients and Methods}

Eligible patients with metastatic, iodine-refractory thyroid carcinoma received sorafenib $400 \mathrm{mg}$ orally twice daily. Responses were measured radiographically every 2 to 3 months. The study end points included response rate, progression-free survival (PFS), and best response by Response Evaluation Criteria in Solid Tumors.

\section{Results}

Thirty patients were entered onto the study and treated for a minimum of 16 weeks. Seven patients (23\%; $95 \% \mathrm{Cl}, 0.10$ to 0.42$)$ had a partial response lasting $18+$ to 84 weeks. Sixteen patients $(53 \%$; $95 \% \mathrm{Cl}, 0.34$ to 0.72 ) had stable disease lasting 14 to $89+$ weeks. Seventeen (95\%) of 19 patients for whom serial thyroglobulin levels were available showed a marked and rapid response in thyroglobulin levels with a mean decrease of $70 \%$. The median PFS was 79 weeks. Toxicity was consistent with other sorafenib trials, although a single patient died of liver failure that was likely treatment related.

\section{Conclusion}

Sorafenib has clinically relevant antitumor activity in patients with metastatic, iodine-refractory thyroid carcinoma, with an overall clinical benefit rate (partial response + stable disease) of $77 \%$, median PFS of 79 weeks, and an overall acceptable safety profile. These results represent a significant advance over chemotherapy in both response rate and PFS and support further investigation of this agent in these patients.

\section{J Clin Oncol 26:4714-4719. (c) 2008 by American Society of Clinical Oncology}

\section{INTRODUCTION}

Thyroid cancer is the most common endocrine malignancy, with 33,500 new cases of thyroid cancers expected to be diagnosed in the United States in 2008. ${ }^{1}$ Differentiated thyroid carcinoma includes papillary and follicular subtypes and comprises $90 \%$ of all cases of thyroid cancer. ${ }^{2}$ Once thyroid cancer metastasizes to distant sites and is no longer amenable to radioactive iodine therapy or surgery, expected survival declines rapidly., ${ }^{3,4}$ The only US Food and Drug Administration-approved therapy for these patients is doxorubicin. ${ }^{5,6}$ In a cooperative group trial of doxorubicin-containing regimens, the progression-free survival (PFS) time of the patients with metastatic, iodine-nonavid, differentiated thyroid cancer was estimated at 2 months, and median overall survival time was 8 months. ${ }^{6}$ Low response rates, short duration of responses, and cardiotoxicity associated with prolonged treatment have rendered doxorubicin a poor option.

Thyroid tumors are highly vascular and overexpress vascular endothelial growth factor (VEGF). ${ }^{7}$ In addition, inhibition of VEGF receptor (VEGFR) signaling has been shown to inhibit growth of thyroid tumors in xenograft models, ${ }^{8}$ thereby providing a strong rationale for targeting VEGFR in this disease. Recent early reports of trials with multikinase inhibitors have shown promise for patients with metastatic thyroid cancer, ${ }^{9-12}$ and most of these compounds under study cause inhibition of VEGFR.

The B-type Raf kinase (BRAF) in the mitogenactivated protein kinase signaling pathway is known to play a key role in thyroid cancer, ${ }^{13}$ and an acidic substitution at a single amino acid residue, V600E, occurs frequently. ${ }^{14} B R A F^{V 600 E}$ is associated with substantially higher basal kinase activity compared with wild-type $B R A F$, and transfection experiments 
have shown that expression of $B R A F^{V 600 E}$ is associated with 70- to 138-fold greater transformation efficacy. ${ }^{14}$ In thyroid cancer, $B R A F^{V 600 E}$ has been found in $29 \%$ to $69 \%$ of papillary thyroid cancers $^{13,15,16}$ and has been associated with aggressive features including extrathyroidal extension and advanced stage. ${ }^{17,18}$ RAS mutations and RET/PTC translocations associated with some thyroid cancers also result in aberrant signaling through BRAF, ${ }^{13,19,20}$ making RAF signaling a logical target in thyroid cancer.

Sorafenib (Nexavar; Onyx Pharmaceuticals, Emeryville, CA, and Bayer Healthcare, Wayne, NJ) is an orally active multi-tyrosine kinase inhibitor with multiple targets, including BRAF, VEGFR1, and VEGFR2. ${ }^{21}$ This drug affects tumor cell proliferation and angiogenesis $^{22}$ and was recently approved by the US Food and Drug Administration for the treatment of renal cell and hepatocellular carcinomas.

Therefore, we conducted a phase II, single-arm trial to determine the efficacy of sorafenib in patients with iodine-refractory metastatic thyroid cancer. This is a report of a planned analysis after treatment of the first 30 patients. Study end points were objective response rate, PFS, and best response, all of which demonstrate the significant clinical activity of sorafenib in this disease.

\section{PATIENTS AND METHODS}

\section{Patients}

Eligible patients were $\geq 18$ years old with metastatic or unresectable thyroid carcinoma for which curative measures were no longer effective. Patients had evidence of measurable disease by Response Evaluation Criteria in Solid Tumors (RECIST). All patients who were enrolled had evidence of disease progression in the year before initiation of treatment. Prior radioactive iodine treatments and one prior biologic treatment (kinase inhibitor, vaccine, or antibody-based therapy) were allowed but not within 3 weeks of treatment. Other eligibility criteria included Eastern Cooperative Oncology Group performance status less than 2, life expectancy more than 3 months, leukocyte count $\geq 3,000 / \mu \mathrm{L}$, absolute neutrophil count $\geq 1,500 / \mu \mathrm{L}$, platelets more than $100,000 / \mu \mathrm{L}$, hemoglobin $\geq 9 \mathrm{~g} / \mathrm{dL}$, serum creatinine $\leq 1.5 \times$ upper limit of normal (ULN) or 24-hour creatinine clearance $\geq 75 \mathrm{~mL} / \mathrm{min}$, serum bilirubin $\leq 1.5 \times \mathrm{ULN}$, serum AST $\leq 2.5 \times \mathrm{ULN}$, alkaline phosphatase $\leq 2.5 \times$ ULN, and prothrombin time-international normalized ratio/partial thromboplastin time $\leq 1.5 \times$ ULN. Premenopausal women were required to have a negative pregnancy test, and all patients of childbearing potential were required to use contraception.

Patients were ineligible if they had previous exposure to a Ras pathway inhibitor (including trastuzumab, epidermal growth factor receptor inhibitors, farnesyl transferase inhibitors, or mitogen-activated ERK kinase inhibitors) or were unable to swallow and retain oral pills. All patients provided written informed consent before enrollment onto the trial. The study protocol was approved by the Institutional Review Board of the University of Pennsylvania.

\section{Study Design}

This was an open-label, single-institution, phase II study of oral sorafenib in patients with metastatic thyroid carcinoma, including differentiated, poorly differentiated, medullary, and anaplastic subtypes. Sorafenib was administered at a dose of $400 \mathrm{mg}$ orally twice a day. A cycle was defined as 4 weeks. Screening evaluations were completed within 2 weeks before the start of study drug, including medical history, demography, review of prior treatment, physical examination, 12-lead ECG, and laboratory evaluations including serum thyroid-stimulating hormone (TSH) and thyroglobulin and urinalysis. Radiologic tests to identify target lesions were performed within 4 weeks of the first dose of study drug.

After beginning treatment, patients were observed at 4-week intervals. At each visit, a history and physical examination were performed, and a $\mathrm{CBC}$, chemistry panel, TSH, and thyroglobulin were drawn. Patients were assessed for new symptoms, compliance with study medications (pill count), and concomitant medications. Dose adjustments were made as needed for toxicity. Response was assessed radiographically after 8 and 16 weeks of treatment and every 12 weeks thereafter.

\section{Study End Points}

The study end points of best objective response rate and stable disease were measured based on the findings on computed tomography (CT) or magnetic resonance imaging using RECIST. Additional end points included best response, duration of response, and time to disease progression (TTP; based on RECIST), clinical progression, or death. Although TTP was the original end point, PFS and TTP were identical in this study, and we have used PFS in this report because it has become the more standard progression end point. All responses were confirmed by a study-designated radiologist. Adverse events were graded with the use of Common Terminology Criteria for Adverse Events (version 3). ${ }^{23}$

\section{Statistical Analysis}

This study was designed to exclude the null hypothesis response rate of $5 \%$ using a Simon two-stage design ${ }^{24}$; the first stage included 30 patients, with an optional second stage including 25 additional patients if at least one response was observed. The observed response rate was tested against the null rate of $5 \%$ using an exact test for binomial proportions. Estimates of PFS (time from starting study drug to progression or death, whichever occurred first) with associated 95\% CIs were obtained using the Kaplan-Meier method. ${ }^{25}$ Comparison of PFS among histologic subtypes was conducted using the logrank test. All enrolled patients who received at least 1 day of treatment were included in this intent-to-treat analysis.

\section{RESULTS}

Between February 28, 2006 and September 13, 2007, 31 patients were entered onto study. Screening failure occurred in one patient as a result of a low WBC count; therefore, the current report includes the first 30 treated patients.

\section{Baseline Characteristics}

Baseline characteristics of the patients entered onto the study are listed in Table 1; five patients had received prior chemotherapy. No patient was previously treated with a kinase inhibitor. All patients had progressive disease on baseline scans before the initiation of treatment, and almost all of our patients (93\%) had uptake of fluorodeoxyglucose on positron emission tomography.

\section{Efficacy}

As of January 19, 2008, the best responses among the 30 patients were assessed (Table 2). This analysis was conducted 4 months after the last patient started treatment. Twenty-five patients were assessable for response, but all 30 patients are included in this intent-to-treat analysis. Responses ranged from progressive disease to a decrease in target lesions of $80 \%$ by RECIST criteria (Fig 1). The objective partial response rate (defined as decrease in sum of longest diameter measurements $\geq 30 \%$ by RECIST) for patients on study was $23 \%$ (seven patients). The exact binomial $95 \%$ CI of $10 \%$ to $42 \%$ excludes the null hypothesis $(P=.0005)$. The stable disease rate (defined as change in sum of longest diameter measurements between $-30 \%$ and $+20 \%$ by RECIST) was 53\% (16 patients; 95\% CI, 34\% to $72 \%$ ). Figure 2 shows the Kaplan-Meier curve for all 30 patients; the median overall PFS time was 79 weeks. Analysis of differentiated thyroid cancers alone revealed a median PFS time of 84 weeks. No patient died before disease progression, and no significant differences in PFS were observed between follicular and papillary subtypes. Seventeen (95\%) of 19 patients for whom serial serum thyroglobulin levels were available 


\begin{tabular}{|c|c|c|c|}
\hline Characteristic & $\begin{array}{c}\text { No. of } \\
\text { Patients } \\
(N=30)\end{array}$ & & $\%$ \\
\hline \multicolumn{4}{|l|}{ Sex } \\
\hline Male & 15 & & 50 \\
\hline Female & 15 & & 50 \\
\hline \multicolumn{4}{|l|}{ Age, years } \\
\hline Mean & \multicolumn{3}{|c|}{63} \\
\hline Range & \multicolumn{3}{|c|}{$31-89$} \\
\hline \multicolumn{4}{|l|}{ Baseline ECOG performance status } \\
\hline 0 & 19 & & 63 \\
\hline 1 & 11 & & 37 \\
\hline \multicolumn{4}{|l|}{ Thyroid cancer subtype } \\
\hline Papillary & 18 & & 60 \\
\hline Follicular/Hürthle cell variant & 9 & & 30 \\
\hline Medullary & 1 & & 3 \\
\hline Poorly differentiated/anaplastic & 2 & & 7 \\
\hline Prior surgery & 30 & & 100 \\
\hline Prior iodine-131 treatment & 28 & & 93 \\
\hline Prior treatment & 5 & & 17 \\
\hline Prior chemotherapy & 3 & & 10 \\
\hline Prior investigational agents & 2 & & 7 \\
\hline Prior external-beam radiation & 11 & & 37 \\
\hline \multicolumn{4}{|l|}{ Prior FDG-PET } \\
\hline FDG-PET scan completed & 29 & & 97 \\
\hline FDG uptake positive & 28 & & 93 \\
\hline
\end{tabular}

Abbreviations: ECOG, Eastern Cooperative Oncology Group; FDG, fluorodeoxyglucose; PET, positron emission tomography.

showed a marked response in thyroglobulin levels, with a mean decrease of $70 \%$ within 4 months of starting treatment.

Two patients with histologically proven poorly differentiated and anaplastic disease had progressive disease as their best response. Of note, the patient with poorly differentiated thyroid cancer had a visible nodule on his shoulder that decreased in size by $50 \%$ at 4 weeks before experiencing progression with new and enlarging pericardial lymph nodes on CT after 7 weeks of treatment. The patient with anaplastic carcinoma had medical complications and rapid clinical progression of her disease within 4 days of beginning sorafenib and was forced to discontinue treatment. Of the 30 patients, five withdrew from the

\begin{tabular}{lcr}
\hline \multicolumn{3}{c}{ Table 2. Patient Response } \\
\hline \multicolumn{1}{c}{ Response } & $\begin{array}{c}\text { No. of } \\
\text { Patients } \\
(\mathrm{N}=30)\end{array}$ & \\
\hline \multicolumn{1}{c}{$\%$} \\
\hline Total assessable patients & 25 & 83 \\
Best response by RECIST & 0 & \\
Complete response & 7 & 23 \\
PR ( $\geq 30 \%)$ & 16 & 53 \\
SD & 23 & 77 \\
Clinical benefit (PR+SD) & 2 & \\
Progressive disease & & 3 \\
Median progression-free survival, weeks & & 79 \\
Overall & & 84 \\
DTC patients
\end{tabular}

Abbreviations: RECIST, Response Evaluation Criteria in Solid Tumors; PR, partial response; SD, stable disease; DTC, differentiated thyroid cancer.

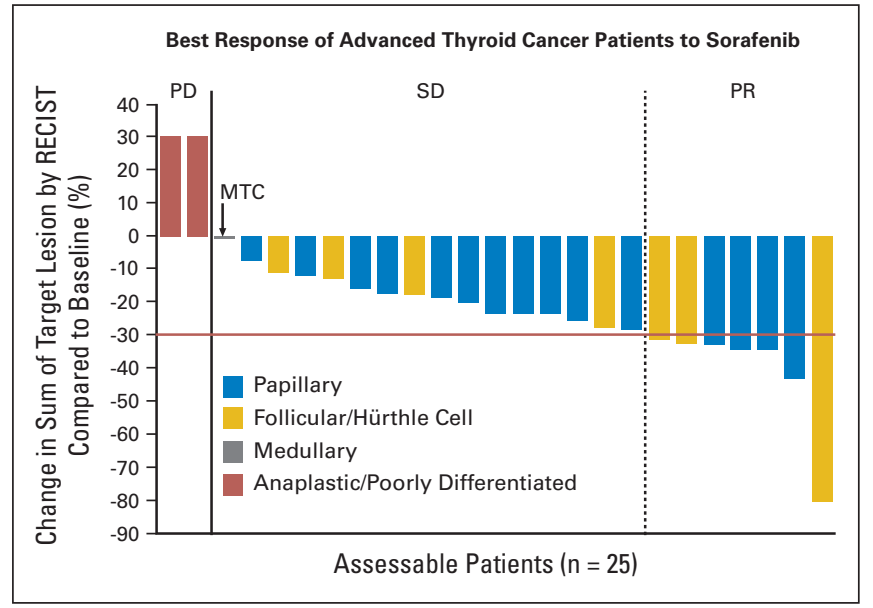

Fig 1. Best overall percentage of change from baseline in target lesion measurement. Baseline radiographic measurements of target lesions were compared with measurements over the course of the study to determine the best change in target lesion size for each patient with data. RECIST, Response Evaluation Criteria in Solid Tumors; PD, progressive disease; SD, stable disease; PR, partial response; MTC, medullary thyroid cancer.

clinical trial before 2 months on study; no imaging response data were available for these patients. Three of these patients withdrew as a result of toxicity, including one patient with a grade 3 rash, one patient with exacerbation of a pre-existing cardiac condition, and one patient with grade 2 fatigue. One patient had a complication related to radiation treatment of a nontarget lesion, and one patient was noncompliant with the study drug. Four other patients on whom scans were available also withdrew. Of these patients, two withdrew because of toxicity from the study drug at 14 and 16 weeks (one patient with medullary thyroid cancer had grade 4 liver toxicity, and the other patient had grade 2 fatigue). One patient with stable disease withdrew after 72 weeks because of pneumonia and resulting fatigue and subsequent decline in performance status, and another patient with stable disease was noncompliant with the study drug at 42 weeks.

The baseline and on-study scans are shown for two patients in Figure 3. The first patient (Fig 3A) had pulmonary metastasis, which resulted in cough and dyspnea on exertion at the time of diagnosis of his primary thyroid cancer. The second patient (Fig 3B) was diagnosed

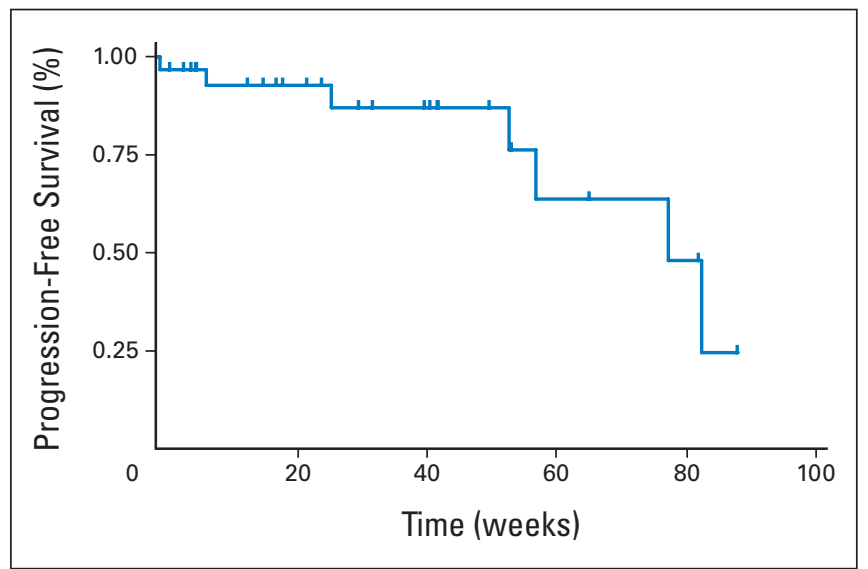

Fig 2. Kaplan-Meier estimate of progression-free survival (PFS) for patients on study. Median PFS was 79.0 weeks. 

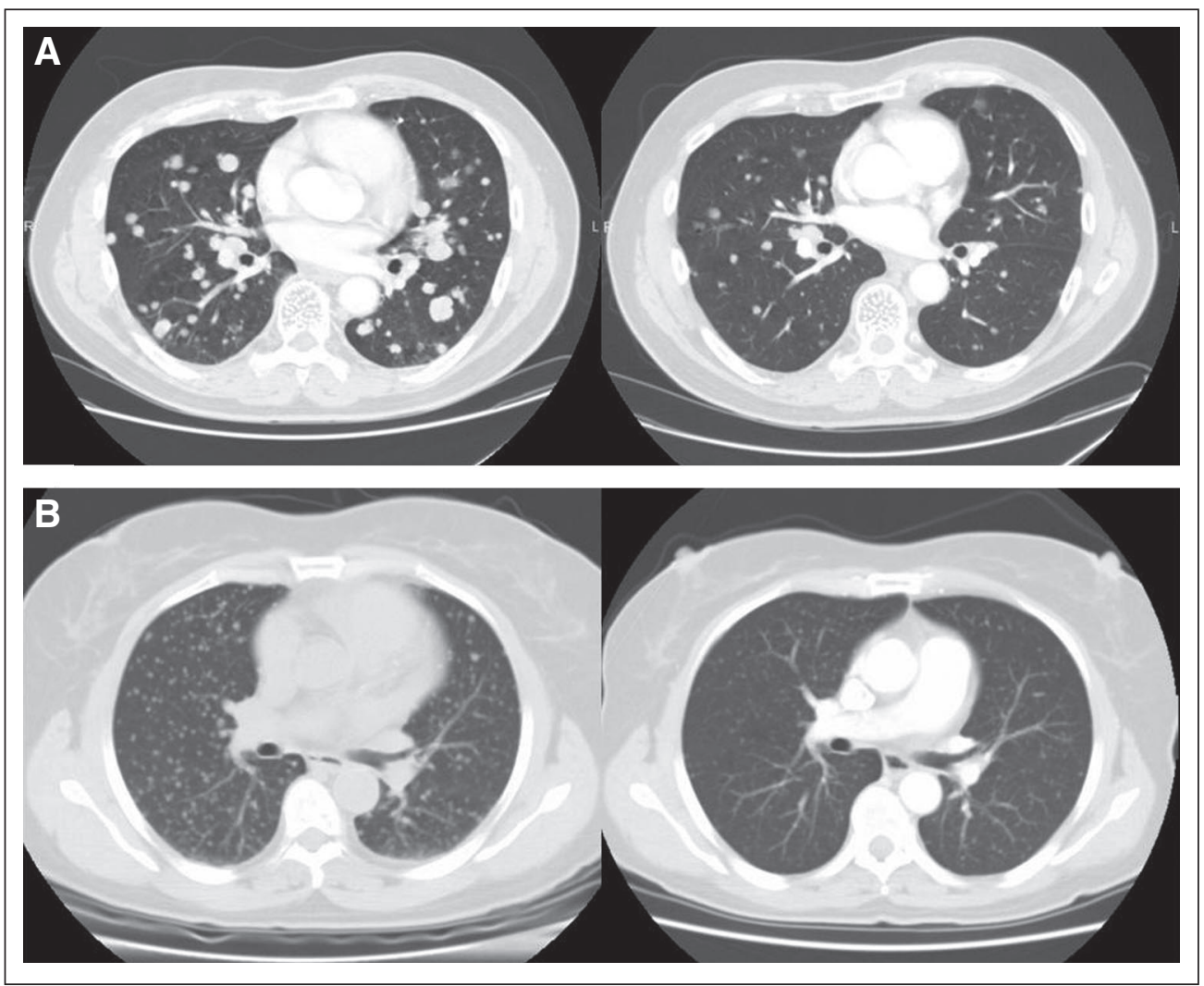

Fig 3. (A) A 60-year-old man with follicular thyroid cancer had metastatic disease in the lung (left). Computed tomography (CT) scans confirm partial response in target lesions (right) after 16 weeks of treatment with sorafenib. (B) A 59-year-old woman with papillary thyroid cancer had widespread miliary lung metastases (left). CT scans show marked improvement in the burden of lung disease after 33 weeks of treatment with sorafenib (right). with papillary thyroid cancer and had undergone thyroidectomy 3 years prior when miliary spread of her thyroid cancer to the lung was discovered. Within a year, the patient began to experience dyspnea on exertion. Both patients noted complete resolution of their symptoms within the first 6 months of treatment with sorafenib.

\section{Adverse Events}

The median duration of treatment was 27 weeks. Six patients (20\%) discontinued treatment as a result of adverse events. Doses were reduced in $47 \%$ of patients (14 patients) to control toxicities. For patients requiring dose reductions, sorafenib was decreased by $25 \%$ (to a total dose of $600 \mathrm{mg}$ once daily) initially, and 10 patients required up to $50 \%$ dose reductions ( $200 \mathrm{mg}$ twice daily) for at least one period to control symptoms. Drug holidays were required as a result of adverse events in $63 \%$ of patients (19 patients). The duration of the dose interruptions ranged from 1 day to 2 weeks, with one patient requiring a 3-week break as a result of musculoskeletal pain. Doses were interrupted most commonly because of fatigue, palmar-plantar erythema, rash, fatigue, stomatitis/mucositis, weight loss, and musculoskeletal pain.

Treatment-related adverse events were predominantly of grade 1 or 2, with the most common events including palmar-plantar erythema, rash, fatigue, stomatitis/mucositis, weight loss, and musculoskeletal pain (Table 3). More severe toxicities observed included hypertension, rash, weight loss, diarrhea, palmar-plantar erythema, fatigue, pruritus, anorexia, anxiety, and elevated lipase and amylase. One patient developed LFT elevations after 8 weeks and, despite dose reductions and cessation of sorafenib per protocol, experienced progression and ultimately died of liver failure 3 months later. Symptoms of palmar- plantar erythema were successfully treated with anti-inflammatory agents, drug interruptions, and dosage reductions. Sixty percent of patients (18 patients) experienced some weight loss. After the degree of weight loss was realized, nutrition counseling and supplementation were started earlier in the treatment course. In six patients, daily megestrol acetate $(800 \mathrm{mg} / \mathrm{d})$ was initiated to improve caloric intake.

Ten patients $(33 \%)$ were referred to their primary endocrinologist for adjustments in their thyroid hormone replacement therapy as a result of an increase in serum TSH to levels greater than $0.10 \mathrm{mU} / \mathrm{L}$ while on sorafenib. One patient had not established a baseline treatment dose before starting the trial, but the remaining nine patients had been on a stable dose before beginning therapy.

\section{DISCUSSION}

Until now, there has been no effective therapy for metastatic thyroid cancer that is not amenable to surgery and that does not concentrate iodine. Response rates with chemotherapy have been so low that best supportive care has been the standard of care for most patients. Both VEGFR and $\mathrm{BRAF}^{\mathrm{V} 600 \mathrm{E}}$ are thought to play a role in the progression of metastatic thyroid cancer. ${ }^{10}$ Recent preliminary results presented suggest the utility of using newer targeted therapies in treating metastatic thyroid cancer. ${ }^{9-12}$ Of the targeted therapies that have recently shown promise in metastatic thyroid cancer, only sorafenib targets both VEGFR and BRAF signaling.

In our trial of sorafenib in patients with metastatic iodinenonavid thyroid cancer, we observed a partial response rate of $23.3 \%$ and a stable disease rate of $53.3 \%$. Median PFS time was estimated to 


\begin{tabular}{|c|c|c|c|c|}
\hline \multirow[b]{2}{*}{ Event } & \multicolumn{2}{|c|}{ Grades 1-2 } & \multicolumn{2}{|c|}{ Grade 3-4 } \\
\hline & $\begin{array}{l}\text { No. of } \\
\text { Patients }\end{array}$ & $\%$ & $\begin{array}{c}\text { No. of } \\
\text { Patients }\end{array}$ & $\%$ \\
\hline \multicolumn{5}{|l|}{ Dermatologic } \\
\hline Rash & 21 & 70 & 3 & 10 \\
\hline Stomatitis/mucositis & 14 & 47 & & \\
\hline Palmar-plantar erythema & 25 & 83 & 3 & 10 \\
\hline Alopecia & 13 & 43 & & \\
\hline Pruritus & 3 & 10 & 1 & 3 \\
\hline \multicolumn{5}{|l|}{ Constitutional } \\
\hline Fatigue & 18 & 60 & 1 & 3 \\
\hline Weight loss & 15 & 50 & 3 & 10 \\
\hline Fever & 2 & 7 & & \\
\hline Anorexia & 5 & 17 & 1 & 3 \\
\hline Musculoskeletal pain & 17 & 57 & & \\
\hline Arthritis & 6 & 20 & & \\
\hline Dehydration & 1 & 3 & & \\
\hline Hoarseness & 6 & 20 & & \\
\hline Epistaxis & 1 & 3 & & \\
\hline Rhinorrhea/URI & 6 & 20 & & \\
\hline Pharyngitis & 5 & 17 & & \\
\hline Xerostomia & 5 & 17 & & \\
\hline Conjunctivitis & 1 & 3 & & \\
\hline Headache & 2 & 7 & & \\
\hline \multicolumn{5}{|l|}{$\mathrm{Gl}$} \\
\hline Diarrhea & 22 & 73 & 2 & 7 \\
\hline Nausea/vomiting & 9 & 30 & & \\
\hline Dyspepsia/Abdominal bloating & 19 & 63 & & \\
\hline Elevated LFTs* & 2 & 7 & 2 & 7 \\
\hline Constipation & 2 & 7 & & \\
\hline Dysphagia & 3 & 10 & & \\
\hline \multicolumn{5}{|l|}{ Pulmonary } \\
\hline Dyspnea/cough & 8 & 27 & & \\
\hline Hemoptysis & 3 & 10 & & \\
\hline \multicolumn{5}{|l|}{ Cardiovascular } \\
\hline Hypertension & 9 & 30 & 4 & 13 \\
\hline Peripheral edema & 1 & 3 & & \\
\hline \multicolumn{5}{|l|}{ Psychological } \\
\hline Depression/mood change & 9 & 30 & & \\
\hline Sleep disturbance/anxiety & 2 & 7 & 1 & 3 \\
\hline Hyperglycemia & 2 & 7 & & \\
\hline \multicolumn{5}{|l|}{ Neurologic } \\
\hline Paresthesias/neuropathy & 6 & 20 & & \\
\hline $\begin{array}{l}\text { Abbreviations: URI, upper respir } \\
{ }^{*} \text { One patient developed grade } 3 / \\
\text { liver failure } 12 \text { weeks later. }\end{array}$ & $\begin{array}{l}\text { y infection; } \\
\text { FT abnorma }\end{array}$ & es at & $\begin{array}{l}\text { function te } \\
\text { weeks and }\end{array}$ & s. \\
\hline
\end{tabular}

be 18 months. The fact that all but two of our patients had fluorodeoxyglucose uptake by their metastatic lesions on positron emission tomography scan highlights the poor prognosis of our patients. ${ }^{26}$ In the Eastern Cooperative Oncology Group trial of doxorubicincontaining regimens, PFS time for patients with metastatic iodinenonavid differentiated thyroid cancer was estimated at 2 months for both arms, and median overall survival time was 8 months. ${ }^{6}$ Thus, our PFS of 18 months may represent a considerable improvement in outcome for these patients. Biologic agents such as sorafenib are associated with prolonged stable disease and modest tumor shrinkage in solid tumors. ${ }^{27,28}$ Patients in whom the disease steadily progresses develop complications that arise from enlarging masses, primarily in the neck and chest. Thus, stable disease results in a clinical benefit for many patients. Our evaluation used RECIST criteria, which were originally developed to assess responses to cytotoxic drugs. Recently, there has been debate over the use of RECIST for biologic agents like sorafenib that provide benefit less with tumor shrinkage than with stable disease. $^{29}$

As is evident on the CT scan in Figure 3B, treatment with sorafenib resulted in a vast improvement in tumor burden in the lung, but because subcentimeter lesions are considered nonmeasurable by RECIST criteria, improvement of this kind is not captured in our overall response rate and points to the need for measures of diseaserelated symptoms and clinical benefit in future studies. Furthermore, as has been documented previously, ${ }^{30}$ treatment with VEGFR tyrosine kinase inhibitors can result in cavitation of lesions with internal necrosis without a change in size of the lesion. In these cases, newer methods for ascertaining decreases in contrast-enhancing tumor, not just tumor diameter, are likely to more accurately reflect antitumor activity that results in disease control.

Although all subtypes of thyroid cancer were included in our study, our patient population consisted mostly of patients with differentiated thyroid cancer; 27 of 30 patients had either papillary or follicular subtypes. Thus, our results may not be generalizable to other subtypes of thyroid cancer. Notably, the two patients who had progressive disease as their best response had poorly differentiated/anaplastic disease.

Several adverse events previously described with sorafenib occurred at similar rates. ${ }^{31}$ The severity of hepatic toxicity in the patient whose liver function continued to deteriorate despite cessation of sorafenib has not been previously reported with sorafenib treatment. The patient refused a liver biopsy, and no infectious cause or drug interaction was identified. Adverse effects were usually manageable with brief holidays from treatment and dose reductions as needed. On reintroduction of the drug, toxicities, if recurrent, were less severe. The frequent interruptions in administration or dose reductions of sorafenib did not prevent most patients from achieving stable disease or a partial response. Because of concern for hypothyroidism in patients treated with other biologic therapies, ${ }^{32}$ we monitored monthly serum TSH in our patients. Because $33 \%$ of the patients on study required alterations in dosing of their thyroid hormone replacement therapy, TSH should be monitored in all thyroid cancer patients receiving sorafenib.

Serum thyroglobulin, a key tumor marker for differentiated thyroid cancers, ${ }^{33}$ decreased precipitously in patients receiving sorafenib. The decrease preceded tumor shrinkage on CT and, therefore, reflects a biologic response in addition to decreased tumor burden. Thus far, we have not been able to elucidate a relationship between thyroglobulin levels and the degree or duration of response. However, secretion of thyroglobulin is likely affected by alterations in cell signaling caused by sorafenib. Therefore, changes in the thyroglobulin levels in the setting of treatment with sorafenib must be interpreted with caution.

A previous trial of sorafenib presented in abstract form in $2006^{34}$ was the first to suggest its activity in patients with iodine-refractory metastatic thyroid cancer. The importance of VEGFR inhibition is evident by the encouraging results of other VEGFR inhibitors in trials for thyroid cancer. ${ }^{9-12}$ Although BRAF signaling (a target of sorafenib) is important in thyroid cancer, its therapeutic significance remains unclear. Genotyping and immunohistochemistry experiments are underway in our laboratory to better elucidate the relationship of BRAF activity and outcome with sorafenib treatment.

In conclusion, long-term disease control in patients with advanced thyroid cancer can be obtained with sorafenib, a well-tolerated 
oral agent. Development of the multikinase inhibitors marks the first significant progress in treating patients with thyroid cancer in more than 30 years. Treatment with sorafenib provided a clinical benefit (partial response + stable disease) rate of $77 \%$ in patients with iodine refractory, metastatic thyroid cancer in whom no other options for treatment were available. These promising results suggest that sorafenib warrants further investigation in the treatment of advanced thyroid cancer.

\section{AUTHORS' DISCLOSURES OF POTENTIAL CONFLICTS OF INTEREST}

Although all authors completed the disclosure declaration, the following author $(s)$ indicated a financial or other interest that is relevant to the subject matter under consideration in this article. Certain relationships marked with a " $U$ " are those for which no compensation was received; those relationships marked with a " $C$ " were compensated. For a detailed description of the disclosure categories, or for more information about ASCO's conflict of interest policy, please refer to the Author Disclosure
Declaration and the Disclosures of Potential Conflicts of Interest section in Information for Contributors.

Employment or Leadership Position: None Consultant or Advisory Role: Keith T. Flaherty, Onyx Pharmaceuticals (C) Stock Ownership: None Honoraria: Keith T. Flaherty, Onyx Pharmaceuticals, Bayer Research Funding: Peter J. O’Dwyer, Onyx Pharmaceuticals, Bayer Expert Testimony: None Other Remuneration: None

\section{AUTHOR CONTRIBUTIONS}

Conception and design: Andrea B. Troxel, Marcia S. Brose

Administrative support: Kathy Ransone

Provision of study materials or patients: Susan J. Mandel, Keith T. Flaherty, Marcia S. Brose

Collection and assembly of data: Vandana Gupta-Abramson, Anoma Nellore, Kanchan Puttaswamy, Maryann Redlinger, Kathy Ransone, Marcia S. Brose Data analysis and interpretation: Vandana Gupta-Abramson, Andrea B. Troxel, Anoma Nellore, Laurie A. Loevner, Peter J. O’Dwyer, Marcia S. Brose Manuscript writing: Vandana Gupta-Abramson, Marcia S. Brose Final approval of manuscript: Vandana Gupta-Abramson, Andrea B. Troxel, Keith T. Flaherty, Peter J. O’Dwyer, Marcia S. Brose

\section{REFERENCES}

1. Jemal $A$, Siegel $R$, Ward $E$, et al: Cancer statistics, 2007. CA Cancer J Clin 57:43-66, 2007

2. Sherman SI: Thyroid carcinoma. Lancet 361: 501-511, 2003

3. Ruegemer JJ, Hay ID, Bergstralh EJ, et al: Distant metastases in differentiated thyroid carcinoma: A multivariate analysis of prognostic variables. J Clin Endocrinol Metab 67:501-508, 1988

4. Shoup M, Stojadinovic A, Nissan A, et al: Prognostic indicators of outcomes in patients with distant metastases from differentiated thyroid carcinoma. J Am Coll Surg 197:191-197, 2003

5. Gottlieb JA, Hill CS Jr: Chemotherapy of thyroid cancer with Adriamycin: Experience with 30 patients. N Engl J Med 290:193-197, 1974

6. Shimaoka K, Schoenfeld DA, DeWys WD, et al: A randomized trial of doxorubicin versus doxorubicin plus cisplatin in patients with advanced thyroid carcinoma. Cancer 56:2155-2160, 1985

7. Tuttle RM, Fleisher M, Francis $G L$, et al: Serum vascular endothelial growth factor levels are elevated in metastatic differentiated thyroid cancer but not increased by short-term TSH stimulation. J Clin Endocrinol Metab 87:1737-1742, 2002

8. Bauer AJ, Terrell R, Doniparthi NK, et al: Vascular endothelial growth factor monoclonal antibody inhibits growth of anaplastic thyroid cancer xenografts in nude mice. Thyroid 12:953-961, 2002

9. Cohen EE, Vokes EE, Rosen LS, et al: A phase II study of axitinib (AG-013736 [AG]) in patients (pts) with advanced thyroid cancers. J Clin Oncol 25: 301s, 2007 (suppl; abstr 6008)

10. Gupta V, Puttaswamy K, Lassoued W, et al: Sorafenib targets BRAF and VEGFR in metastatic thyroid carcinoma. J Clin Oncol 25:303s, 2007 (suppl; abstr 6019)

11. Sherman SI, Schlumberger MJ, Droz J, et al: Initial results from a phase II trial of motesanib diphosphate (AMG 706) in patients with differentiated thyroid cancer (DTC). J Clin Oncol 25:303s, 2007 (suppl; abstr 6017)
12. Wells SA, Gosnell JE, Gagel RF, et al: Vandetanib in metastatic hereditary medullary thyroid cancer: Follow-up results of an open-label phase II trial. J Clin Oncol 25:303s, 2007 (suppl; abstr 6018)

13. Cohen $Y$, Xing $M$, Mambo $E$, et al: BRAF mutation in papillary thyroid carcinoma. J Natl Cancer Inst 95:625-627, 2003

14. Davies H, Bignell GR, Cox C, et al: Mutations of the BRAF gene in human cancer. Nature 417:949954, 2002

15. Namba $H$, Nakashima $M$, Hayashi $T$, et al: Clinical implication of hot spot BRAF mutation, V599E, in papillary thyroid cancers. J Clin Endocrinol Metab 88:4393-4397, 2003

16. Kim KH, Kang DW, Kim SH, et al: Mutations of the BRAF gene in papillary thyroid carcinoma in a Korean population. Yonsei Med J 45:818-821, 2004

17. Xing M: BRAF mutation in thyroid cancer. Endocr Relat Cancer 12:245-262, 2005

18. Xing $M$, Westra $W H$, Tufano RP, et al: BRAF mutation predicts a poorer clinical prognosis for papillary thyroid cancer. J Clin Endocrinol Metab 90:6373-6379, 2005

19. Nikiforova MN, Caudill CM, Biddinger $P$, et al: Prevalence of RET/PTC rearrangements in Hashimoto's thyroiditis and papillary thyroid carcinomas. Int J Surg Pathol 10:15-22, 2002

20. Ciampi R, Nikiforov YE: RET/PTC rearrangements and BRAF mutations in thyroid tumorigenesis. Endocrinology 148:936-941, 2007

21. Wilhelm SM, Carter $C$, Tang $L$, et al: BAY 43-9006 exhibits broad spectrum oral antitumor activity and targets the RAF/MEK/ERK pathway and receptor tyrosine kinases involved in tumor progression and angiogenesis. Cancer Res 64:7099-7109, 2004

22. Murphy DA, Makonnen S, Lassoued W, et al: Inhibition of tumor endothelial ERK activation, angiogenesis, and tumor growth by sorafenib (BAY439006). Am J Pathol 169:1875-1885, 2006

23. National Cancer Institute: Common Terminology Criteria for Adverse Events, v. 3.0. http://ctep.cancer.gov/reporting/ctc.html
24. Simon R: Optimal two-stage designs for phase II clinical trials. Control Clin Trials 10:1-10, 1989

25. Kaplan EL, Meier P: Nonparametric estimation from incomplete observations. J Am Stat Assoc 53:457-481, 1958

26. Robbins RJ, Wan Q, Grewal RK, et al: Realtime prognosis for metastatic thyroid carcinoma based on 2-[18F]fluoro-2-deoxy-D-glucose-positron emission tomography scanning. J Clin Endocrinol Metab 91:498-505, 2006

27. Yang JC, Haworth L, Sherry RM, et al: A randomized trial of bevacizumab, an anti-vascular endothelial growth factor antibody, for metastatic renal cancer. N Engl J Med 349:427-434, 2003

28. Ratain MJ, Flaherty KT, Stadler WM, et al, Preliminary antitumor activity of BAY 43-9006 in metastatic renal cell carcinoma and other advanced refractory solid tumors in a phase II randomized discontinuation trial. J Clin Oncol 23:381s, 2004 (suppl; abstr 4501)

29. Ratain MJ, Eckhardt SG: Phase II studies of modern drugs directed against new targets: If you are fazed, too, then resist RECIST. J Clin Oncol 22:4442-4445, 2004

30. Sun S, Schiller JH: Angiogenesis inhibitors in the treatment of lung cancer. Crit Rev Oncol Hematol 62:93-104, 2007

31. Escudier B, Eisen T, Stadler WM, et al: Sorafenib in advanced clear-cell renal-cell carcinoma. N Engl J Med 356:125-134, 2007

32. Desai J, Yassa L, Marqusee E, et al: Hypothyroidism after sunitinib treatment for patients with gastrointestinal stromal tumors. Ann Intern Med 145:660-664, 2006

33. Duren $M$, Siperstein $A E$, Shen $W$, et al: Value of stimulated serum thyroglobulin levels for detecting persistent or recurrent differentiated thyroid cancer in high- and low-risk patients. Surgery 126 : 13-19, 1999

34. Kloos RMR, Knopp M, Heverhagen J, et al: Significant clinical and biologic activity of RAF/ VEGF-R kinase inhibitor BAY 43-9006 in patients with metastatic papillary thyroid carcinoma (PTC): Updated results of a phase II study. J Clin Oncol 24:288s, 2006 (suppl; abstr 5534)

\section{Acknowledgment}

The authors are grateful to patients and for the ongoing support from the Templeton Family Thyroid Cancer Research Fund. 